\title{
Faint variable stars observed with Kepler
}

\author{
Catherine Lovekin ${ }^{1, \star}$ and Jasmin Tompkins ${ }^{1}$ \\ ${ }^{1}$ Physics Department, Mount Allison University, Sackville, NB, E4L 1C6, Canada
}

\begin{abstract}
We present preliminary analysis of approximately 10 variable stars observed with Kepler. The sample stars are faint, and have temperatures greater than $8000 \mathrm{~K}$. The stars were observed for up to three quarters (Q14-Q16) in long cadence mode. Frequencies were extracted with Period04, and 1-21 frequencies were detected in each quarter, with an average of 8 frequencies per quarter. Some variability is detected from quarter to quarter, while the dominant frequencies remain unchanged. We fit the frequencies using MESA models between 1.5 and 3 solar masses, and varied the core overshoot. Best fitting properties of each of these stars will be discussed.
\end{abstract}

\section{Introduction}

$\delta$ Scuti stars are typically between 1.4 and $2.5 M_{\odot}$, and are found in the HR diagram where the classical instability strip intersects the main sequence. As such, they are expected to be found in the transition region between stars with convective envelopes and radiative cores $\left(<1.5 M_{\odot}\right)$ and stars with radiative envelopes and convective cores $\left(>1.5 M_{\odot}\right)$. At the same time, $\delta$ Scuti stars are often rapidly rotating ([1]). This makes $\delta$ Scuti stars ideal for constraining the effects of rotation and convective overshoot.

\section{Frequency analysis}

We selected ten stars with listed temperatures greater than $8000 \mathrm{~K}$ in the Kepler input catalogue. These stars were previously known to be variable ([2]), but are quite faint, from 14th to 16th magnitude. These stars were observed from Q14 to Q16, and most of the stars have continuous coverage across all three quarters. We used Period04 ([3]) to analyse the light curves and extract the frequencies. Since these stars are quite faint, and the noise level is correspondingly high, only a few frequencies were detected in each star with SNR $>4$. Across all quarters, we were able to extract between 1 and 21 frequencies per star. We detected some quarter-to-quarter variability, but the dominant frequency remains unchanged in all stars. A sample Fourier spectrum is shown for KIC 9818160 in Figure 1.

The observed frequencies are primarily greater than $5 \mathrm{c} / \mathrm{d}$, which suggests these stars are primarily $\delta$ Scuti stars. Uncertainties in the frequencies, amplitudes, and phases were calculated in Period04 using 500 iterations of a Monte Carlo simulation. At the present time, we have not made any attempt to search for patterns in the observed frequencies. With as few as 3 or 4 observed modes in some of the stars, it may not be possible to measure large frequency separations.

^clovekin@mta.ca 


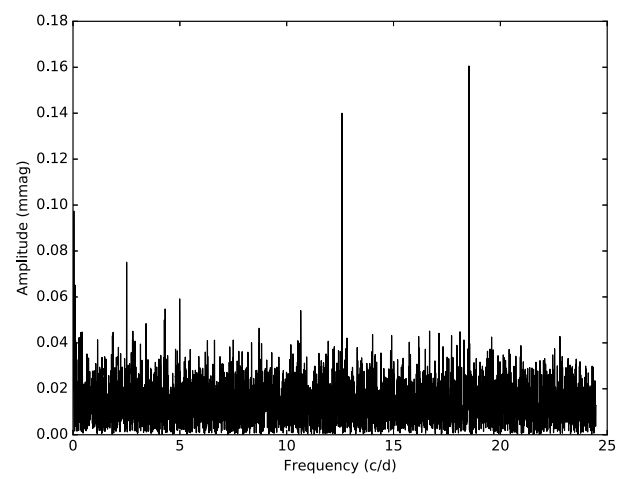

Figure 1. The raw Fourier spectrum for KIC 9818160. Four frequencies with SNR $>4$ were detected in this star.

\section{Models}

Preliminary fits to the observed frequencies were calculated using models calculated with MESA ([4]) and GYRE ([5]). The model grid extended from 2.0 to $2.8 M_{\odot}$ in steps of $0.05 M_{\odot}$. We also calculated a few models outside the main grid at 1.5 and $3.2 M_{\odot}$. We also varied the convective core overshoot of these models, varying $f_{o v}$ from 0.1 to 0.2 in steps of 0.02 . The observed frequencies were directly compared to the model frequencies via a $\chi^{2}$ algorithm. The best fitting models cover the full range of our model grid. No obvious trends were discovered in either mass or convective core overshoot. However, the best fitting models were universally young, with core hydrogen fractions from 0.69 to 0.59 .

\section{Conclusion}

We present preliminary modelling of 10 faint variable stars observed with Kepler. The observed frequencies are consistent with $\delta$ Scuti pulsations, as expected based on the parameters of these stars in the KIC. More detailed fitting of these stars, including a larger model grid is in progress. We also plan to expand our grid by including the effects of rotation as well as convective overshoot.

Acknowledgments: This paper includes data collected by the Kepler mission as part of the Guest Observer program. Funding for the Kepler mission is provided by the NASA Science Mission Directorate. This research has made use of NASA's Astrophysics Data System and Astropy, a community developed core Python package for astronomy.

\section{References}

[1] Breger, M., Communications in Asteroseismology, 150, 25 (2007)

[2] Kinemuchi, K., Still, M., Fanelli, M., \& Kepler Science, Bulletin of the American Astronomical Society, 43, 201.06 (2011)

[3] Lenz, P., \& Breger, M., Communications in Asteroseismology, 146, 53 (2005)

[4] Paxton, B., Bildsten, L., Dotter, A., et al., ApJS, 192, 3 (2011)

[5] Townsend, R. H. D., \& Teitler, S. A., MNRAS, 435, 3406 (2013) 